17. Chuang, J.Z., Zhao, Y., and Sung, C.H. 2007. SARAregulated vesicular targeting underlies formation of the light-sensing organelle in mammalian rods. Cell. 130:535-547.

18. Townes-Anderson, E. 1995. Intersegmental fusion in vertebrate rod photoreceptors. Rod cell structure revisited. Invest. Ophthalmol. Vis. Sci. 36:1918-1933.

19. Andrews, L.D., and Cohen, A.I. 1983. Freeze-fracture studies of photoreceptor membranes: new observations bearing upon the distribution of cholesterol. J. Cell Biol. 97:749-755.

20. Riedl, J., et al. 2008. Lifeact: a versatile marker to visualize F-actin. Nat. Methods. doi:10.1038/ nmeth.1220.

21. Smith, L.M., et al. 2008. CD133/prominin-1 is a potential therapeutic target for antibody-drug conjugates in hepatocellular and gastric cancers. Br. J. Cancer. doi:10.1038/sj.bjc.6604437.

\title{
Marking a path to transplant tolerance
}

\author{
Vicki Seyfert-Margolis ${ }^{1,2}$ and Laurence A. Turka ${ }^{2,3}$
}

\begin{abstract}
${ }^{1}$ Department of Medicine, University of California, San Francisco, San Francisco, California, USA. 2Immune Tolerance Network, Bethesda, Maryland, USA. ${ }^{3}$ Department of Medicine, University of Pennsylvania School of Medicine, Philadelphia, Pennsylvania, USA.
\end{abstract}

\begin{abstract}
Long-term allograft survival requires lifelong immunosuppression, which comes with serious side effects. Inducing immune tolerance to the transplant would enable immunosuppression withdrawal and revolutionize the quality of life of transplant recipients. In this issue of the JCI, MartinezLlordella et al. identify a profile of biomarkers that predict tolerance in liver transplant recipients (see the related article beginning on page 2845). These findings translate into a new means for prospectively selecting liver transplant patients who would benefit from immunosuppression withdrawal and ultimately may guide development of tolerogenic therapies that allow for allograft acceptance without the use of long-term immunosuppression.
\end{abstract}

The road to solid organ transplant tolerance may be somewhat shortened with the discovery of several new biomarkers for tolerance, as reported by Martínez-Llordella et al. in this issue of the JCI (1). These studies represent a significant advance in the ongoing effort to wean liver transplant recipients off immunosuppressive drugs. While newer immunosuppression protocols have vastly improved acute rejection rates in solid organ transplantation over the past 20 years, success is still not without its price - the consequences of longterm immunosuppression, often resulting in renal toxicity, opportunistic infections, and/or lymphoproliferative disease, remain significant clinical concerns. Clearly then, the idea that select liver transplant recipients might withdraw from all immunosuppression with little or no risk to their allograft deserves attention.

\section{Tolerance occurs in liver transplantation}

The liver has long been appreciated to be a relatively immunoprivileged organ. For example, in some rodent models of transplantation, liver grafts are often spontaneously accepted without a need for any

Conflict of interest: L.A. Turka has equity in GlaxoSmithKline.

Citation for this article: J. Clin. Invest. 118:2684-2686 (2008). doi:10.1172/JCI36552. immunosuppression, and it has been proposed that unique populations of antigenpresenting cells and ECs that reside in the liver are responsible for this phenomenon $(2,3)$. Studies of liver transplant recipients dating back to 1997 demonstrated that small numbers of patients could cease all immunosuppressive medications and still maintain a healthy graft (4). This observation triggered a series of studies in which the prospective withdrawal of immunosuppression was attempted in small cohorts of liver transplant recipients. The first such study was performed at the University of Pittsburgh, where 19\% of patients $(n=37)$ became drug free for at least 1 year (5). Subsequent studies in Japan and in the United Kingdom revealed that prospective weaning could be achieved in approximately $20 \%$ of enrolled patients. In these cases, weaning was successful in a total of 18 patients in the United Kingdom (6) and in a larger number in Japan $(7,8)$. More recently, the Immune Tolerance Network has established its support of a study of immunosuppression withdrawal in pediatric, parent-to-child living-donor liver transplant recipients. Overall, these studies and others have led to the generally accepted estimate that $20 \%$ of livertransplanted patients may be successfully withdrawn from immunosuppression.

That $20 \%$ of patients might be spared the risks of long-term immunosuppres- sion compels us to ask the question, can we identify these subjects a priori? One might similarly ask, can we do better than $20 \%$ ? The answer to both of these questions lies in defining biomarkers that indicate a propensity for successful immunosuppression withdrawal and that more clearly define the state of allograft tolerance.

\section{Can we predict tolerance?}

In their current study, Martínez-Llordella, et al. appear to have taken us a major step forward by providing a relatively small set of robust markers that can distinguish tolerant from nontolerant liver transplant recipients and from healthy individuals (1). Using a combination of quantitative real-time PCR and flow cytometry techniques, the authors point to increases in the numbers of peripheral T cells using the $\gamma \delta$ antigen receptor, in particular those expressing the $\delta 1$ form of the receptor, and to a difference in the activation state of circulating NK cells, as shown by a small set of differentially expressed genes. Critically, the predictive biomarkers were derived using a training set of samples and then validated in an independently gathered cohort of test-set patients. This work complements previously published reports of increased $\delta 1-\gamma \delta \mathrm{TCR}^{+} \mathrm{T}$ cells in the blood of tolerant liver transplant patients $(7,9,10)$, thereby giving further credence to this measure as a valid biomarker of tolerance in this setting. Importantly, given the relative ease with which these assays can be performed and their targeted list of differentially expressed genes (26 in total), these assessments can be easily validated for use in the clinic (Figure 1).

\section{Do predictive markers tell us about mechanism?}

The proposed biomarkers (1) also raise important biological questions: What do the results from tolerant liver transplant 


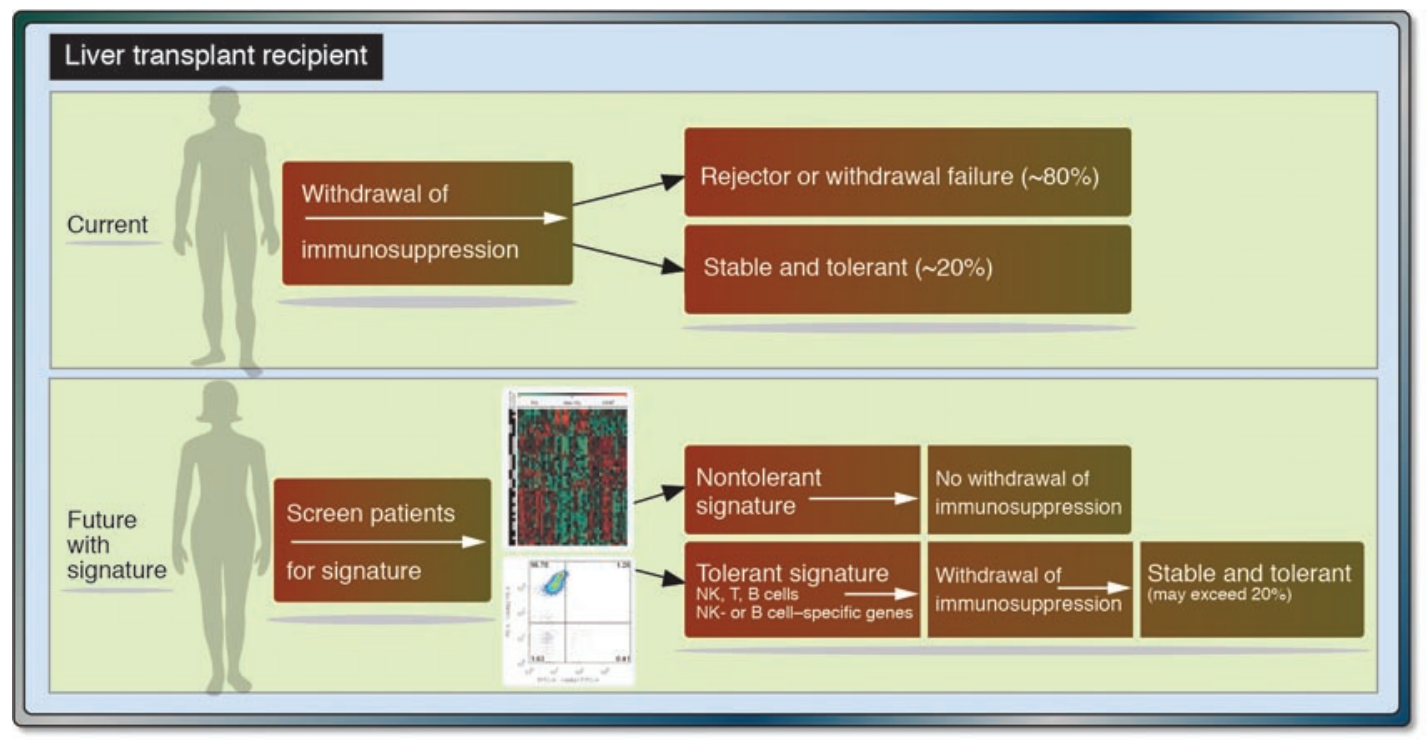

\section{Figure 1}

Schema illustrating how potential signatures of tolerance in liver transplant recipients can be utilized. In their current study in this issue of the $\mathrm{JCl}$, Martínez-Llordella et al. (1) have defined new markers in the peripheral blood of liver transplant patients that correlate with a state of tolerance. This finding has significant implications for the clinic, where these signatures can serve as a screening tool for patient management. Liver transplant recipients on standard-of-care immunosuppression can be screened for these defined tolerance signatures based on expressed gene profiles and cell subsets, as measured by microarray, quantitative real-time PCR, and flow cytometry. If patients score positively for the "protolerance" signature, a program of immunosuppression withdrawal would be initiated, leading to a potential increase in the proportion of liver transplant recipients who can live free of immunosuppressive drugs, thereby improving long-term outcomes and quality of life.

patients tell us about how tolerance is achieved? The increased number of $\gamma \delta \mathrm{TCR}^{+}$ $\mathrm{T}$ cells, particularly $\delta 1-\gamma \delta \mathrm{TCR}^{+} \mathrm{T}$ cells, in the peripheral blood points to a redistribution of cells that are normally resident in the liver and intestinal epithelium $(2,3)$. Since the liver is also known to be a major site of $\gamma \delta \mathrm{TCR}^{+} \mathrm{T}$ cell development $(2,3$, 11 ), increased extrathymic development of these cells, in particular $\delta 1-\gamma \delta \mathrm{TCR}^{+}$ $\mathrm{T}$ cells, may be induced by the initial inflammatory response elicited by the transplant. $\delta 1-\gamma \delta \mathrm{TCR}^{+} \mathrm{T}$ cells also compose the major subset of intestinal intraepithelial lymphocytes, again another potential site of extrathymic development (3). These $\gamma \delta \mathrm{TCR}^{+} \mathrm{T}$ cells may have a tolerogenic capacity, as their expressed gene profile (confirmed by direct protein staining) was specifically altered in tolerant patients studied by Martínez-Llordella et al. (1), with induction of molecules that may mark regulatory function. Other evidence for immune regulation in the tolerant patients studied here includes the elevated levels of $\mathrm{CD} 25^{+} \mathrm{FoxP}^{+}{ }^{+} \mathrm{CD} 4^{+}$Tregs.

NK cells are another important part of the story. Both their numbers and their activation status differ markedly between tolerant and healthy controls or nontolerant liver transplant patients (1). Tolerant patients had activated NK cell-signaling pathways, members of which included a number of proinflammatory genes. Activated NK cells have been shown in liver infection and injury models to play a crucial role in recruitment of cells to the liver through a multistep cytokine/chemokine cascade $(12,13)$. They also have been shown to modulate liver injury by balancing Th1 proinflammatory and Th2 antiinflammatory responses (2). NKT cells, also part of the tolerant signature presented here, are believed to be antiinflammatory, as they have been shown to secrete IL-4 and have been protective in some animal models of autoimmunity $(2,11,14)$.

While the report by Martínez-Llordella et al. in this issue (1) paints a picture of tolerance at the systemic level by focusing on peripheral blood, it is likely that the "real action" happens in the liver. This is a microenvironment that is known to promote tolerance, a situation that most likely evolved to avoid responses to the continuous onslaught of foreign food and bacterial antigens that enter via blood draining from the intestines $(2,3)$. In this environment, NK cells recruit $T$ cells to the liver, where they encounter antigens in the presence of liver sinusoidal ECs $(2,3)$. Liver sinusoidal ECs promote the development of $\mathrm{CD}^{+}$ $\mathrm{T}$ cells into regulatory, IL-4-, or IL-10-pro- ducing cell types $(3,15)$. In addition, $\mathrm{CD}^{+}$ $\mathrm{T}$ cells also may be induced to undergo apoptosis when primed in the liver (3, 16). In fact, the genes encoding TP53 and CDKN1A, both associated with induced cell death $(17,18)$, are among those found to be elevated in the peripheral blood of the tolerant liver transplant recipients in this study. Nonetheless, these ideas, while appealing, remain associations, and their ultimate relationship to immune privilege within the liver or to tolerance to liver transplants remains speculative.

Also unknown is the extent to which the tolerance signature identified by Martínez-Llordella et al. (1) can be extended to recipients of other types of organ and tissue grafts. It is possible that the biomarkers are "universal" for transplant tolerance but more likely that differences in organs, treatment regimens, and degrees of tissue compatibility will alter the profile of tolerance in individual patients, as is suggested by emerging studies in tolerant renal transplant recipients $(19,20)$.

Regardless of mechanism, MartínezLlordella, et al. (1) have taken important steps forward in making transplant tolerance a clinical reality for a large subset of liver transplant patients. With these biomarkers in hand, prospective immunosuppression 
withdrawal studies may employ more personalized, science-based decision making, stratifying patients according to validated assessments for their propensity for tolerance. As well, these findings provide a basis for new studies in both humans and animal models to better understand solid organ transplant tolerance and the important and unique contributions that the liver, an immune organ itself, makes to the tolerance process following liver transplantation.

Address correspondence to: Vicki Seyfert-Margolis, Immune Tolerance Network, 3 Bethesda Metro Center, Suite 400, Bethesda, Maryland 20814, USA. Phone: (240) 235-6133; Fax: (240) 235-6198; E-mail: vseyfert@immunetolerance.org.

1. Martínez-Llordella, M., et al. 2008. Using transcriptional profiling to develop a diagnostic test of operational tolerance in liver transplant recipients. J. Clin. Invest. 118:2845-2857.

2. Racanelli, V., and Rehermann, B. 2006. The liver as an immunological organ. Hepatology. 43:S54-S62.

3. Crispe, I.N. 2003. Hepatic T cells and liver toler- ance. Nat. Rev. Immunol. 3:51-62.

4. Starzl, T.E., et al. 1993. Cell migration and chimerism after whole-organ transplantation: the basis of graft acceptance. Hepatology. 17:1127-1152.

5. Mazariegos, G.V., et al. 1997. Weaning of immunosuppression in liver transplant recipients. Transplantation. 63:243-249.

6. Devlin, J., et al. 1998. Defining the outcome of immunosuppression withdrawal after liver transplantation. Hepatology. 27:926-933.

7. Koshiba, T., et al. 2007. Clinical, immunological, and pathological aspects of operational tolerance after pediatric living-donor liver transplantation. Transpl. Immunol. 17:94-97.

8. Takatsuki, M., et al. 2001. Analysis of alloreactivity and intragraft cytokine profiles in living donor liver transplant recipients with graft acceptance. Transpl. Immunol. 8:279-286.

9. Li, Y., et al. 2004. Analyses of peripheral blood mononuclear cells in operational tolerance after pediatric living donor liver transplantation. Am. J. Transplant. 4:2118-2125.

10. Martínez-Llordella, M., et al. 2007. Multiparameter immune profiling of operational tolerance in liver transplantation. Am. J. Transplant. 7:309-319.

11. Abo, T., Kawamura, T., and Watanabe, H. 2000. Physiological responses of extrathymic T cells in the liver. Immunol. Rev. 174:135-149.

12. McIntyre, K.W., and Welsh, R.M. 1986. Accumulation of natural killer and cytotoxic T large granular lymphocytes in the liver during virus infection. J. Exp. Med. 164:1667-1681.
13. Toyabe, S., et al. 1997. Requirement of IL-4 and liver NK1+ T cells for concanavalin A-induced hepatic injury in mice. J. Immunol. 159:1537-1542.

14. Vicari, A.P., Mocci, S., Openshaw, P., O'Garra, A., and Zlotnik, A. 1996. Mouse gamma delta $\mathrm{TCR}+\mathrm{NK} 1.1+$ thymocytes specifically produce interleukin-4, are major histocompatibility complex class I independent, and are developmentally related to alpha beta TCR+NK1.1+ thymocytes. Eur. J. Immunol. 26:1424-1429.

15. Knolle, P.A., et al. 1999. Induction of cytokine production in naive CD4(+) T cells by antigen-presenting murine liver sinusoidal endothelial cells but failure to induce differentiation toward Th1 cells. Gastroenterology. 116:1428-1440.

16. Limmer, A., et al. 2000. Efficient presentation of exogenous antigen by liver endothelial cells to CD8+ T cells results in antigen-specific T-cell tolerance. Nat. Med. 6:1348-1354.

17. Levine, A.J. 1997. p53, the cellular gatekeeper for growth and division. Cell. 88:323-331.

18. Levkau, B., et al. 1998. Cleavage of p21Cip1/Waf1 and p27Kip1 mediates apoptosis in endothelial cells through activation of $\mathrm{Cdk} 2$ : role of a caspase cascade. Mol. Cell. 1:553-563.

19. Kawai, T., et al. 2008. HLA-mismatched renal transplantation without maintenance immunosuppression. N. Engl. J. Med. 358:353-361.

20. Brouard, S., et al. 2007. Identification of a peripheral blood transcriptional biomarker panel associated with operational renal allograft tolerance. Proc. Natl. Acad. Sci. U. S. A. 104:15448-15453.

\title{
Immune response hinders therapy for lysosomal storage diseases
}

\author{
Katherine P. Ponder \\ Department of Internal Medicine and Department of Biochemistry and Molecular Biophysics, \\ Washington University School of Medicine, St. Louis, Missouri, USA.
}

\begin{abstract}
Enzyme replacement therapy (ERT) for the lysosomal storage disease mucopolysaccharidosis I (MPS I) involves i.v. injection of $\alpha$-L-iduronidase, which can be taken up by cells throughout the body. While a significant immune response to ERT has been shown in patients with MPS I, little is known about what effect anti-enzyme antibodies have on treatment efficacy. In this issue of the JCI, Dickson et al. demonstrate that anti-enzyme antibodies inhibit enzyme uptake and substantially limit the therapeutic efficacy of ERT in canines with MPS I (see the related article beginning on page 2868). Furthermore, the induction of immune tolerance - via oral delivery of cyclosporine $A$ and azathioprine for two months at the time of initiation of ERT with recombinant human $\alpha$-L-iduronidase - improved enzyme uptake in organs. Therefore, transient immunosuppression may enhance ERT for lysosomal storage diseases.
\end{abstract}

Nonstandard abbreviations used: ERT, enzyme replacement therapy; GAG, glycosaminoglycan; IDUA, $\alpha$-L-iduronidase; LSD, lysosomal storage disease; M6P, mannose 6-phosphate; MPS I, mucopolysaccharidosis I.

Conflict of interest: The author has declared that no conflict of interest exists.

Citation for this article: J. Clin. Invest. 118:2686-2689 (2008). doi:10.1172/JCI36521.
Intravenous injection of recombinant proteins can treat genetic deficiencies such as lysosomal storage diseases (LSD) or hemophilia. Since the patient does not produce the normal protein, the therapeutic protein can be recognized as foreign and induce antibodies. It has been known for decades that antibodies that inhibit the coagula- tion function of Factor VIII develop in $30 \%$ of patients with severe hemophilia A (1). These antibodies are known as inhibitors and bind to epitopes of Factor VIII that are very important for its coagulation function. However, it has been less clear how antibodies may affect the efficacy of recombinant proteins that are injected i.v. to treat LSDs. The study by Dickson et al. in this issue of the JCI (2) demonstrates that antibodies reduce the efficacy of this therapeutic approach for the LSD mucopolysaccharidosis I (MPS I) and identifies an immunosuppressive regimen that is highly effective at blocking antibody formation when given at the time of initiation of protein therapy in dogs with MPS I.

\section{Enzyme replacement therapy for LSDs}

LSDs are due to a deficiency in any of several enzymes that degrade various sub- 